\title{
EXISTENCE AND UNIQUENESS OF SOLUTIONS FOR DELAY STOCHASTIC EVOLUTION EQUATIONS
}

\author{
Tomás CARABALLO, María J. GARRIDO-ATIENZA and José REAL \\ Dpto. de Ecuaciones Diferenciales y Análisis Numérico, \\ Universidad de Sevilla, \\ Apdo. de Correos 1160, \\ 41080-Sevilla, Spain. \\ e-mails: caraball@cica.es ; mgarrido@numer.us.es; real@numer.us.es
}

\begin{abstract}
Some results on the existence and uniqueness of solutions for stochastic evolution equations containing some hereditary characteristics are proved. In fact, our theory is developed from a variational point of view and in a general functional setting which permit us to deal with several kinds of delay terms in a unified formulation.
\end{abstract}

\section{Introduction and statement of the problem}

When one wants to model some evolution phenomena arising in physics, biology, engineering, etc., some hereditary characteristics such as after-effect, time-lag, time-delay can appear in the variables. Typical examples can be found in the researches of materials with termal memory, biochemical reactions, population models, etc. (see, for instance, Ruess [10], Wu [11] and references cited therein). This enables us to think that the problem could be better modeled by considering a functional differential equation which takes into account the history of the system. However, in most cases, some kind of randomness can appear in the problem, so that the system should be modeled by a stochastic form of the functional equation. Motivated by these facts, our main purpose in this paper is to analyse the existence and uniqueness of solutions for a class of nonlinear stochastic PDEs with time delays in a variational context, which, in particular, extend and complete the results in Caraballo [2] and Caraballo et al. [4].

Firt of all, we would like to mention that, in the deterministic framework, there exists a wide literature on the existence of different kind of solutions (strong, mild, integral, etc.) to functional differential equations even in the more general context of differential inclusions. It is well worth reading the work by Ruess [10] where one can find a description of the different techniques used to handle this question, in addition to a large list of references concerning these methods (e.g. method of lines, Galerkin approximations, Kato approximants, etc.). However, from a variational point of view, only a few works have been published (Artola [1] for linear and semilinear retarded equations, Caraballo [3] for a more general nonlinear monotone situation in the functional framework, among others).

As for the stochastic problem in the variational setting, even much less has been done. As far as we know, only the works by Real [8,9] (in the linear case), Caraballo [2] (nonlinear problem with variable delay) and Caraballo et al. [4] have appeared up to date. But, as the assumptions in these works are rather restrictive so that the operators involved in the equations cannot be general enough, we are now interested in developing a theory which, in particular, contains the previous works and which permits us to prove existence and uniqueness of solution for a wider class of systems. 
To start off, let us state the abstract framework in which our analysis will be carried out. Let $V$ and $H$ be two real separable Hilbert spaces such that

$$
V \subset H \equiv H^{*} \subset V^{*}
$$

where the injections are continuous and dense.

We denote by $\|\cdot\|,|\cdot|$ and $\|\cdot\|_{*}$ the norms in $V, H$ and $V^{*}$ respectively; by $((\cdot, \cdot))$ and $(\cdot, \cdot)$ the scalar products in $V$ and $H$ respectively; and by $\langle\cdot, \cdot\rangle$ the duality product between $V^{*}$ and $V$.

Assume that $\{\Omega, \mathcal{F}, P\}$ is a complete probability space, equipped with a normal filtration $\left\{\mathcal{F}_{t}\right\}_{t \geq 0}$, i.e., $\mathcal{F}_{0}$ contains all $A \in \mathcal{F}$ such that $P(A)=0$ and $\mathcal{F}_{t}=\bigcap_{s>t} \mathcal{F}_{s}, \forall t \geq 0$. Denote $\mathcal{F}_{t}=\mathcal{F}_{0}$ for all $t \leq 0$.

We suppose also given $\{W(t)\}_{t \geq 0}$, a real valued $\left\{\mathcal{F}_{t}\right\}-$ Wiener process.

Given real numbers $a<b$, and a separable Hilbert space $\mathcal{H}$ we will denote by $I^{2}(a, b ; \mathcal{H})$ the space of all processes $X \in L^{2}(\Omega \times(a, b), \mathcal{F} \otimes \mathcal{B}((a, b)), d P \otimes d t ; \mathcal{H})$ (where $\mathcal{B}((a, b))$ denotes the Borel $\sigma$-algebra on $(a, b))$ such that $X(t)$ is $\mathcal{F}_{t}$-measurable a.e. $t \in(a, b)$. The space $I^{2}(a, b ; \mathcal{H})$ is a closed subspace of $L^{2}(\Omega \times(a, b), \mathcal{F} \otimes \mathcal{B}((a, b)), d P \otimes d t ; \mathcal{H})$.

We will denote by $C(a, b ; \mathcal{H})$ the Banach space of all continuous functions from $[a, b]$ into $\mathcal{H}$ equipped with sup norm. We will write $L^{2}(\Omega ; C(a, b ; \mathcal{H}))$ instead of $L^{2}(\Omega, \mathcal{F}, d P ; C(a, b ; \mathcal{H}))$.

Let us also consider two fixed real numbers $T>0$ and $h>0$. If we consider a function $x \in C(-h, T ; \mathcal{H})$, for each $t \in[0, T]$ we will denote by $x_{t} \in C(-h, 0 ; \mathcal{H})$ the function defined by $x_{t}(s)=x(t+s) \forall s \in[-h, 0]$. Moreover, if $y \in L^{2}(-h, T ; \mathcal{H})$, we will also denote by $y_{t} \in L^{2}(-h, 0 ; \mathcal{H})$, for a.e. $t \in(0, T)$, the function defined by $y_{t}(s)=y(t+s)$ a.e. $s \in(-h, 0)$.

Let $A(t, \cdot): V \rightarrow V^{*}$ be a family of nonlinear operators defined a.e. $t \in(0, T)$ and satisfying:

(A.1) (Measurability) $\forall v \in V$, the map $t \in(0, T) \rightarrow A(t, v) \in V^{*}$ is Lebesgue measurable.

(A.2) (Hemicontinuity) the map

$$
\theta \in \mathbb{R} \rightarrow\langle A(t, u+\theta v), w\rangle \in \mathbb{R}
$$

is continuous $\forall u, v, w \in V$, and a.e. $t \in(0, T)$.

(A.3) (Boundedness) there exists $c>0$ such that $\|A(t, v)\|_{*} \leq c\|v\| \quad \forall v \in V$, a.e. $t \in$ $(0, T)$.

(A.4) (Monotonicity and Coercivity): there exist $\alpha>0$ and $\lambda \in \mathbb{R}$ such that

$$
-2\langle A(t, u)-A(t, v), u-v\rangle+\lambda|u-v|^{2} \geq \alpha\|u-v\|^{2}, \quad \forall u, v \in V \text {, a.e. } t \in(0, T) .
$$

Let $F_{1}:(0, T) \times C(-h, 0 ; H) \rightarrow V^{*}$ and $F_{2}:(0, T) \times C(-h, 0 ; V) \rightarrow V^{*}$ be two families of nonlinear operators defined a.e. $t \in(0, T)$ such that:

$\left(F_{1} .1\right) \forall \xi \in C(-h, 0 ; H)$, the map $t \in(0, T) \longmapsto F_{1}(t, \xi) \in V^{*}$ is Lebesgue measurable,

$\left(F_{1} .2\right) F_{1}(t, 0)=0$, a.e. $t \in(0, T)$,

$\left(F_{1} .3\right)$ there exists $C_{F_{1}}>0$ such that 
$\left\|F_{1}(t, \xi)-F_{1}(t, \eta)\right\|_{*}^{2} \leq C_{F_{1}}|\xi-\eta|_{C(-h, 0 ; H)}^{2}, \quad \forall \xi, \eta \in C(-h, 0 ; H)$, a.e. $t \in(0, T)$,

$\left(F_{2} .1\right) \forall \xi \in C(-h, 0 ; V)$, the map $t \in(0, T) \longmapsto F_{2}(t, \xi) \in V^{*}$ is Lebesgue measurable,

$\left(F_{2} .2\right) F_{2}(t, 0)=0$, a.e. $t \in(0, T)$,

$\left(F_{2} .3\right)$ there exists $C_{F_{2}}>0$ such that

$$
\left\|F_{2}(t, \xi)-F_{2}(t, \eta)\right\|_{*}^{2} \leq C_{F_{2}}\|\xi-\eta\|_{C(-h, 0 ; V)}^{2}, \forall \xi, \eta \in C(-h, 0 ; V) \text {, a.e. } t \in(0, T),
$$

$\left(F_{2} .4\right)$ there exists $K_{F_{2}}>0$ such that $\forall x, y \in C(-h, T ; V)$, and $\forall t \in[0, T]$,

$$
\int_{0}^{t}\left\|F_{2}\left(s, x_{s}\right)-F_{2}\left(s, y_{s}\right)\right\|_{*}^{2} d s \leq K_{F_{2}} \int_{-h}^{t}\|x(s)-y(s)\|^{2} d s .
$$

Let also $G_{0}:(0, T) \times C(-h, 0 ; H) \rightarrow H$ and $G_{1}:(0, T) \times C(-h, 0 ; V) \rightarrow H$ be another two families of nonlinear operators defined a.e. $t \in(0, T)$ such that:

$\left(G_{0} .1\right) \forall \xi \in C(-h, 0 ; H)$, the map $t \in(0, T) \longmapsto G_{0}(t, \xi) \in H$ is Lebesgue measurable,

$\left(G_{0} .2\right) G_{0}(t, 0)=0$, a.e. $t \in(0, T)$,

$\left(G_{0} .3\right)$ there exists $C_{G_{0}}>0$ such that

$$
\left|G_{0}(t, \xi)-G_{0}(t, \eta)\right|^{2} \leq C_{G_{0}}|\xi-\eta|_{C(-h, 0 ; H)}^{2}, \quad \forall \xi, \eta \in C(-h, 0 ; H) \text {, a.e. } t \in(0, T),
$$

$\left(G_{1} .1\right) \forall \xi \in C(-h, 0 ; V)$, the map $t \in(0, T) \longmapsto G_{1}(t, \xi) \in H$ is Lebesgue measurable,

$\left(G_{1} .2\right) G_{1}(t, 0)=0$, a.e. $t \in(0, T)$,

$\left(G_{1} .3\right)$ there exists $C_{G_{1}}>0$ such that

$$
\left|G_{1}(t, \xi)-G_{1}(t, \eta)\right|^{2} \leq C_{G_{1}}\|\xi-\eta\|_{C(-h, 0 ; V)}^{2}, \forall \xi, \eta \in C(-h, 0 ; V) \text {, a.e. } t \in(0, T),
$$

$\left(G_{1} .4\right)$ there exists $K_{G_{1}}>0$ such that $\forall x, y \in C(-h, T ; V)$, and $\forall t \in[0, T]$,

$$
\int_{0}^{t}\left|G_{1}\left(s, x_{s}\right)-G_{1}\left(s, y_{s}\right)\right|^{2} d s \leq K_{G_{1}} \int_{-h}^{t}\|x(s)-y(s)\|^{2} d s .
$$

We consider the problem 


$$
\left\{\begin{aligned}
u \in I^{2}(-h, T ; V) \cap L^{2}(\Omega ; C(-h, T ; H)), & \\
u(t)= & \psi(0)+\int_{0}^{t} A(s, u(s)) d s+\int_{0}^{t}\left(F_{1}\left(s, u_{s}\right)+F_{2}\left(s, u_{s}\right)+f(s)\right) d s \\
& \quad+\int_{0}^{t}\left(G_{0}\left(s, u_{s}\right)+G_{1}\left(s, u_{s}\right)+g(s)\right) d W(s), t \in[0, T], \\
u(t)= & \psi(t), t \in[-h, 0],
\end{aligned}\right.
$$

where $f \in I^{2}\left(0, T ; V^{*}\right), g \in I^{2}(0, T ; H)$ and $\psi \in I^{2}(-h, 0 ; V) \cap L^{2}(\Omega ; C(-h, 0 ; H))$ are given.

Remark 1.1. It is not difficult to deduce from $\left(F_{1} .1\right)-\left(F_{1} .3\right)$ that if $u \in I^{2}(-h, T ; V) \cap$ $L^{2}(\Omega ; C(-h, T ; H))$, the process $F_{1}\left(t, u_{t}\right)$ belongs to $I^{2}\left(0, T ; V^{*}\right)$. Also, by means of $\left(G_{0} .1\right)$ $\left(G_{0} .3\right)$, the process $G_{0}\left(t, u_{t}\right)$ belongs to $I^{2}(0, T ; H)$.

Remark 1.2. Observe that by $\left(F_{2} \cdot 1\right)-\left(F_{2} .3\right)$, for a given $x \in C(-h, T ; V)$, the function $F_{2}^{x}:(0, T) \rightarrow V^{*}$ defined by $F_{2}^{x}(t)=F_{2}\left(t, x_{t}\right)$ a.e.t $\in(0, T)$, belongs to $L^{2}\left(0, T ; V^{*}\right)$. Then, thanks to $\left(F_{2} .4\right)$, the mapping

$$
\Xi: x \in C(-h, T ; V) \mapsto F_{2}^{x} \in L^{2}\left(0, T ; V^{*}\right)
$$

has a unique extension to a mapping $\widetilde{\Xi}$ which is uniformly continuous from $L^{2}(-h, T ; V)$ into $L^{2}\left(0, T ; V^{*}\right)$. From now on, we will also write $F_{2}\left(t, x_{t}\right)=\widetilde{\Xi}(x)(t)$ for each $x \in L^{2}(-h, T ; V)$, and for every $x, y \in L^{2}(-h, T ; V)$ it holds

$$
\int_{0}^{t}\left\|F_{2}\left(s, x_{s}\right)-F_{2}\left(s, y_{s}\right)\right\|_{*}^{2} d s \leq K_{F_{2}} \int_{-h}^{t}\|x(s)-y(s)\|^{2} d s \quad \forall t \in[0, T] .
$$

By a similar argument, we can define $G_{1}\left(t, x_{t}\right) \in L^{2}(0, T ; H)$ for each $x \in L^{2}(-h, T ; V)$, and $\forall x, y \in L^{2}(-h, T ; V)$ it follows

$$
\int_{0}^{t}\left|G_{1}\left(s, x_{s}\right)-G_{1}\left(s, y_{s}\right)\right|^{2} d s \leq K_{G_{1}} \int_{-h}^{t}\|x(s)-y(s)\|^{2} d s \quad \forall t \in[0, T] .
$$

Thus, if $u \in I^{2}(-h, T ; V)$ is given, the process $F_{2}\left(t, u_{t}\right)$ belongs to $I^{2}\left(0, T ; V^{*}\right)$, the process $G_{1}\left(t, u_{t}\right)$ belongs to $I^{2}(0, T ; H)$, and, consequently, $\forall u, v \in I^{2}(-h, T ; V)$ we obtain

$$
\int_{0}^{t}\left\|F_{2}\left(s, u_{s}\right)-F_{2}\left(s, v_{s}\right)\right\|_{*}^{2} d s \leq K_{F_{2}} \int_{-h}^{t}\|u(s)-v(s)\|^{2} d s \quad \forall t \in[0, T], P-a . s .
$$

and

$$
\int_{0}^{t}\left|G_{1}\left(s, u_{s}\right)-G_{1}\left(s, v_{s}\right)\right|^{2} d s \leq K_{G_{1}} \int_{-h}^{t}\|u(s)-v(s)\|^{2} d s \quad \forall t \in[0, T], P-a . s . .
$$

As a consequence of the preceding remarks, the terms appearing in problem $(P)$ make sense. Now, we are interested in establishing some results on the existence and uniqueness of solution to $(P)$ under some additional assumptions. To this respect, it is worth mentioning that in the absence of hereditary characteristics (i.e. when $h=0$ ), our problem has been solved by Pardoux [6] (see also Da Prato and Zabczyk [5] for a different approach); in the linear case 
containing variable delays, it has also been treated by Real [9]; Caraballo [2] considered the nonlinear monotone situation with variable delay but for bounded operators $F_{i}$ and $G_{i}$, and finally, Caraballo et al. [4] provided an answer to our problem in the particular situations in which $F_{2} \equiv 0, F_{1}(t, \cdot)$ is a family of operators from $V$ into $H$ and, what is more important, under stronger assumptions on the family of operators which do not allow us to cover a wide class of applications (e.g. in the case of unbounded operators, essentially the ones containing distributed delays satisfy the assumptions in [4]). Thus, on the one hand, the results we shall obtain can be considered as extensions to the nonlinear case of those obtained in Real [9]. On the other hand, the presence of the term $F_{1}$ and the hypotheses that we shall impose on $F_{2}$ and $G_{1}$, permit us, as we have already mentioned, to treat examples which cannot be handled with the results in Caraballo et al. [4].

The paper is organized as follows. In Section 2, we prove a first result on the existence and uniqueness of solution for the problem $(P)$ in the particular case $F_{2} \equiv G_{1} \equiv 0$. Then, in Section 3, we establish an existence and uniqueness result for the complete problem. Finally, an example is considered in the last Section to illustrate our results.

\section{A first existence and uniqueness result}

In this section, we shall consider the problem

$$
\left\{\begin{aligned}
u \in I^{2}(-h, T ; V) \cap L^{2}(\Omega ; C(-h, T ; H)), & \\
u(t)= & \psi(0)+\int_{0}^{t} A(s, u(s)) d s+\int_{0}^{t}\left(F_{1}\left(s, u_{s}\right)+f(s)\right) d s \\
& \quad+\int_{0}^{t}\left(G_{0}\left(s, u_{s}\right)+g(s)\right) d W(s), t \in[0, T], \\
u(t)= & \psi(t), t \in[-h, 0] .
\end{aligned}\right.
$$

We can now prove the following result:

Theorem 2.1 Assume that hypotheses $(A .1)-(A .4),\left(F_{1} .1\right)-\left(F_{1} .3\right)$ and $\left(G_{0} .1\right)-\left(G_{0} .3\right)$ hold. Then, for every $\psi \in I^{2}(-h, 0 ; V) \cap L^{2}(\Omega ; C(-h, 0 ; H)), f \in I^{2}\left(0, T ; V^{*}\right)$ and $g \in I^{2}(0, T ; H)$, there exists a unique solution $u$ to the problem $\left(P^{\prime}\right)$.

Proof.

Uniqueness of solutions. Assume that $u, v \in I^{2}(-h, T ; V) \cap L^{2}(\Omega ; C(-h, T ; H))$ are two solutions of $\left(P^{\prime}\right)$. Then, Itô's formula and condition (A.4) imply that for all $t \in[0, T]$

$$
\begin{aligned}
|u(t)-v(t)|^{2}+ & \alpha \int_{0}^{t}\|u(s)-v(s)\|^{2} d s \\
\leq & \lambda \int_{0}^{t}|u(s)-v(s)|^{2} d s \\
& +2 \int_{0}^{t}\left\langle F_{1}\left(s, u_{s}\right)-F_{1}\left(s, v_{s}\right), u(s)-v(s)\right\rangle d s \\
& +2 \int_{0}^{t}\left(G_{0}\left(s, u_{s}\right)-G_{0}\left(s, v_{s}\right), u(s)-v(s)\right) d W(s) \\
& +\int_{0}^{t}\left|G_{0}\left(s, u_{s}\right)-G_{0}\left(s, v_{s}\right)\right|^{2} d s .
\end{aligned}
$$


Therefore,

$$
\begin{aligned}
E\left[\sup _{0 \leq s \leq t}|u(s)-v(s)|^{2}\right]+\alpha E \int_{0}^{t}\|u(s)-v(s)\|^{2} d s \\
\leq 2|\lambda| E \int_{0}^{t}|u(s)-v(s)|^{2} d s+2 E \int_{0}^{t}\left|G_{0}\left(s, u_{s}\right)-G_{0}\left(s, v_{s}\right)\right|^{2} d s \\
\quad+4 E \int_{0}^{t}\left\|F_{1}\left(s, u_{s}\right)-F_{1}\left(s, v_{s}\right)\right\|_{*}\|u(s)-v(s)\| d s \\
\quad+4 E\left[\sup _{0 \leq s \leq t} \int_{0}^{s}\left(G_{0}\left(\theta, u_{\theta}\right)-G_{0}\left(\theta, v_{\theta}\right), u(\theta)-v(\theta)\right) d W(\theta)\right]
\end{aligned}
$$

for all $t \in[0, T]$. Now, we can estimate the terms on the right-hand side of (2.1). On the one hand,

$$
\begin{aligned}
4 E & \int_{0}^{t}\left\|F_{1}\left(s, u_{s}\right)-F_{1}\left(s, v_{s}\right)\right\|_{*}\|u(s)-v(s)\| d s \\
& \leq E \int_{0}^{t}\left[\frac{8}{\alpha}\left\|F_{1}\left(s, u_{s}\right)-F_{1}\left(s, v_{s}\right)\right\|_{*}^{2}+\frac{\alpha}{2}\|u(s)-v(s)\|^{2}\right] d s \\
& \leq \frac{8}{\alpha} C_{F_{1}} E \int_{0}^{t}\left|u_{s}-v_{s}\right|_{C(-h, 0 ; H)}^{2} d s+\frac{\alpha}{2} E \int_{0}^{t}\|u(s)-v(s)\|^{2} d s \\
& \leq \frac{8}{\alpha} C_{F_{1}} E \int_{0}^{t} \sup _{0 \leq r \leq s}|u(r)-v(r)|^{2} d s+\frac{\alpha}{2} E \int_{0}^{t}\|u(s)-v(s)\|^{2} d s .
\end{aligned}
$$

On the other hand, Burkholder-Davis-Gundy's inequality yields that

$$
\begin{aligned}
4 E & {\left[\sup _{0 \leq s \leq t} \int_{0}^{s}\left(G_{0}\left(\theta, u_{\theta}\right)-G_{0}\left(\theta, v_{\theta}\right), u(\theta)-v(\theta)\right) d W(\theta)\right] } \\
& \leq 12 E\left\{\sup _{0 \leq s \leq t}|u(s)-v(s)|\left[\int_{0}^{t}\left|G_{0}\left(\theta, u_{\theta}\right)-G_{0}\left(\theta, v_{\theta}\right)\right|^{2} d \theta\right]^{\frac{1}{2}}\right\} \\
& \leq \frac{1}{2} E\left(\sup _{0 \leq s \leq t}|u(s)-v(s)|^{2}\right)+72 E \int_{0}^{t}\left|G_{0}\left(\theta, u_{\theta}\right)-G_{0}\left(\theta, v_{\theta}\right)\right|^{2} d \theta \\
& \leq \frac{1}{2} E\left(\sup _{0 \leq s \leq t}|u(s)-v(s)|^{2}\right)+72 C_{G_{0}} E \int_{0}^{t}\left|u_{\theta}-v_{\theta}\right|_{C(-h, 0 ; H)}^{2} d \theta \\
& \leq \frac{1}{2} E\left(\sup _{0 \leq s \leq t}|u(s)-v(s)|^{2}\right)+72 C_{G_{0}} E \int_{0}^{t} \sup _{0 \leq r \leq \theta}|u(r)-v(r)|^{2} d \theta .
\end{aligned}
$$

Thus, (2.1)-(2.3) imply that for all $t \in[0, T]$

$$
\begin{aligned}
\frac{1}{2} E & {\left[\sup _{0 \leq s \leq t}|u(s)-v(s)|^{2}\right]+\frac{\alpha}{2} E \int_{0}^{t}\|u(s)-v(s)\|^{2} d s } \\
& \leq\left[2|\lambda|+\frac{8}{\alpha} C_{F_{1}}+74 C_{G_{0}}\right] E \int_{0}^{t} \sup _{0 \leq r \leq \theta}|u(r)-v(r)|^{2} d \theta .
\end{aligned}
$$

Now, uniqueness follows immediately from Gronwall's lemma. 
Existence of solutions: We denote $u^{0} \equiv 0$, and define by recurrence a sequence $\left\{u^{n}\right\}_{n \geq 1}$ of processes as solutions to the problem

$$
\left\{\begin{array}{l}
u^{n} \in I^{2}(-h, T ; V) \cap L^{2}(\Omega ; C(-h, T ; H)), \\
u^{n}(t)=\psi(0)+\int_{0}^{t}\left(A\left(s, u^{n}(s)\right)-\frac{\lambda}{2} u^{n}(s)\right) d s+\frac{\lambda}{2} \int_{0}^{t} u^{n-1}(s) d s \\
+\int_{0}^{t}\left(F_{1}\left(s, u_{s}^{n-1}\right)+f(s)\right) d s+\int_{0}^{t}\left(G_{0}\left(s, u_{s}^{n-1}\right)+g(s)\right) d W(s), t \in[0, T], \\
u^{n}(t)=\psi(t), t \in[-h, 0] .
\end{array}\right.
$$

Observe that $u^{0} \equiv 0 \in I^{2}(-h, T ; V) \cap L^{2}(\Omega ; C(-h, T ; H))$, and by Remark 1.1, if $u^{n-1} \in$ $I^{2}(-h, T ; V) \cap L^{2}(\Omega ; C(-h, T ; H))$, it follows that $F_{1}\left(t, u_{t}^{n-1}\right) \in I^{2}\left(0, T ; V^{*}\right)$, and $G_{0}\left(t, u_{t}^{n-1}\right) \in$ $I^{2}(0, T ; H)$, and consequently, from the results in Pardoux [6], there exists a unique $u^{n} \in$ $I^{2}(-h, T ; V) \cap L^{2}(\Omega ; C(-h, T ; H))$ which is a solution of $\left(P_{n}^{\prime}\right)$.

Now, we want to prove that $\left\{u^{n}\right\}_{n>1}$ converges in $I^{2}(-h, T ; V) \cap L^{2}(\Omega ; C(-h, T ; H))$ to a process $u$ which will be the solution of problem $\left(P^{\prime}\right)$.

Applying Itô's formula to the process $u^{n+1}(t)-u^{n}(t), n \geq 1$, and using condition (A.4), we obtain

$$
\begin{aligned}
\left|u^{n+1}(t)-u^{n}(t)\right|^{2}+ & \alpha \int_{0}^{t}\left\|u^{n+1}(s)-u^{n}(s)\right\|^{2} d s \\
\leq & \lambda \int_{0}^{t}\left(u^{n+1}(s)-u^{n}(s), u^{n}(s)-u^{n-1}(s)\right) d s \\
& +2 \int_{0}^{t}\left\langle F_{1}\left(s, u_{s}^{n}\right)-F_{1}\left(s, u_{s}^{n-1}\right), u^{n+1}(s)-u^{n}(s)\right\rangle d s \\
& +2 \int_{0}^{t}\left(G_{0}\left(s, u_{s}^{n}\right)-G_{0}\left(s, u_{s}^{n-1}\right), u^{n+1}(s)-u^{n}(s)\right) d W(s) \\
& +\int_{0}^{t}\left|G_{0}\left(s, u_{s}^{n}\right)-G_{0}\left(s, u_{s}^{n-1}\right)\right|^{2} d s
\end{aligned}
$$

for all $t \in[0, T]$.

Consequently, (2.4) yields

$$
\begin{aligned}
& E\left[\sup _{0 \leq s \leq t}\left|u^{n+1}(s)-u^{n}(s)\right|^{2}\right]+\alpha E \int_{0}^{t}\left\|u^{n+1}(s)-u^{n}(s)\right\|^{2} d s \\
& \leq 2|\lambda| E \int_{0}^{t}\left|u^{n+1}(s)-u^{n}(s)\right|\left|u^{n}(s)-u^{n-1}(s)\right| d s \\
&+4 E \int_{0}^{t}\left|\left\langle F_{1}\left(s, u_{s}^{n}\right)-F_{1}\left(s, u_{s}^{n-1}\right), u^{n+1}(s)-u^{n}(s)\right\rangle\right| d s \\
&+4 E\left[\sup _{0 \leq s \leq t} \int_{0}^{s}\left(G_{0}\left(\theta, u_{\theta}^{n}\right)-G_{0}\left(\theta, u_{\theta}^{n-1}\right), u^{n+1}(\theta)-u^{n}(\theta)\right) d W(\theta)\right] \\
&+2 E \int_{0}^{t}\left|G_{0}\left(s, u_{s}^{n}\right)-G_{0}\left(s, u_{s}^{n-1}\right)\right|^{2} d s .
\end{aligned}
$$

Now, observe that 


$$
\begin{aligned}
2|\lambda| E & \int_{0}^{t}\left|u^{n+1}(s)-u^{n}(s)\right|\left|u^{n}(s)-u^{n-1}(s)\right| d s \\
& \leq 2 \beta|\lambda| E \int_{0}^{t}\left\|u^{n+1}(s)-u^{n}(s)\right\|\left|u^{n}(s)-u^{n-1}(s)\right| d s \\
& \leq \frac{\alpha}{3} E \int_{0}^{t}\left\|u^{n+1}(s)-u^{n}(s)\right\|^{2} d s+\frac{3 \lambda^{2} \beta^{2}}{\alpha} E \int_{0}^{t} \sup _{0 \leq \theta \leq s}\left|u^{n}(\theta)-u^{n-1}(\theta)\right|^{2} d s
\end{aligned}
$$

where $\beta>0$ is a constant such that $|v| \leq \beta\|v\|, \forall v \in V$.

On the other hand, thanks to condition $\left(F_{1} .3\right)$, we can obtain

$$
\begin{aligned}
4 E & \int_{0}^{t}\left|\left\langle F_{1}\left(s, u_{s}^{n}\right)-F_{1}\left(s, u_{s}^{n-1}\right), u^{n+1}(s)-u^{n}(s)\right\rangle\right| d s \\
& \leq 4 E \int_{0}^{t}\left\|F_{1}\left(s, u_{s}^{n}\right)-F_{1}\left(s, u_{s}^{n-1}\right)\right\|_{*}\left\|u^{n+1}(s)-u^{n}(s)\right\| d s \\
& \leq E \int_{0}^{t}\left[\frac{12}{\alpha}\left\|F_{1}\left(s, u_{s}^{n}\right)-F_{1}\left(s, u_{s}^{n-1}\right)\right\|_{*}^{2}+\frac{\alpha}{3}\left\|u^{n+1}(s)-u^{n}(s)\right\|^{2}\right] d s \\
& \leq \frac{12}{\alpha} C_{F_{1}} E \int_{0}^{t}\left|u_{s}^{n}-u_{s}^{n-1}\right|_{C(-h, 0 ; H)}^{2} d s+\frac{\alpha}{3} E \int_{0}^{t}\left\|u^{n+1}(s)-u^{n}(s)\right\|^{2} d s \\
& \leq \frac{12}{\alpha} C_{F_{1}} E \int_{0}^{t} \sup _{0 \leq r \leq s}\left|u^{n}(r)-u^{n-1}(r)\right|^{2} d s+\frac{\alpha}{3} E \int_{0}^{t}\left\|u^{n+1}(s)-u^{n}(s)\right\|^{2} d s .
\end{aligned}
$$

In a similar manner as for uniqueness, we can obtain from Burkholder-Davis-Gundy's inequality that

$$
\begin{aligned}
4 E & {\left[\sup _{0 \leq s \leq t} \int_{0}^{s}\left(G_{0}\left(\theta, u_{\theta}^{n}\right)-G_{0}\left(\theta, u_{\theta}^{n-1}\right), u^{n+1}(\theta)-u^{n}(\theta)\right) d W(\theta)\right] } \\
& \leq \frac{1}{2} E\left(\sup _{0 \leq s \leq t}\left|u^{n+1}(s)-u^{n}(s)\right|^{2}\right)+72 C_{G_{0}} E \int_{0}^{t} \sup _{0 \leq r \leq \theta}\left|u^{n}(r)-u^{n-1}(r)\right|^{2} d \theta .
\end{aligned}
$$

Then, we can get from (2.5)-(2.8) and $\left(G_{0} .3\right)$, that there exists a positive constant $k$ such that for all $n \geq 1$ and all $t \in[0, T]$

$$
\begin{gathered}
\left.\frac{1}{2} E\left[\sup _{0 \leq s \leq t}\left|u^{n+1}(s)-u^{n}(s)\right|^{2}\right]+\frac{\alpha}{3} E \int_{0}^{t} \| u^{n+1}(s)-u^{n}(s)\right) \|^{2} d s \\
\leq \frac{k}{2} E \int_{0}^{t} \sup _{0 \leq r \leq \theta}\left|u^{n}(r)-u^{n-1}(r)\right|^{2} d \theta .
\end{gathered}
$$

Now, we define

$\left.\rho^{n}(t)=\frac{1}{2} E\left[\sup _{0 \leq s \leq t}\left|u^{n+1}(s)-u^{n}(s)\right|^{2}\right]+\frac{\alpha}{3} E \int_{0}^{t} \| u^{n+1}(s)-u^{n}(s)\right) \|^{2} d s, \forall n \geq 1, \forall t \in[0, T]$.

Then, (2.9) immediately implies that

$$
\rho^{n}(t) \leq k \int_{0}^{t} \rho^{n-1}(s) d s, \forall n \geq 1, \forall t \in[0, T]
$$


and, consequently, by iterating the preceding inequality, we obtain

$$
\rho^{n}(t) \leq \frac{k^{n-1} T^{n-1}}{(n-1) !} \rho^{1}(T), \forall n \geq 1, \forall t \in[0, T] .
$$

Since $u^{n+1}(t)=u^{n}(t), \forall t \in[-h, 0],(2.10)$ implies that $\left\{u^{n}\right\}_{n>1}$ is a Cauchy sequence in $I^{2}(-h, T ; V) \cap L^{2}(\Omega ; C(-h, T ; H))$. Thus, there exists $u \in I^{2}(-h, T ; V) \cap L^{2}(\Omega ; C(-h, T ; H))$ such that

$$
u^{n} \rightarrow u \text { in } I^{2}(-h, T ; V) \cap L^{2}(\Omega ; C(-h, T ; H)) .
$$

Thanks to conditions $\left(F_{1} .3\right)$ and $\left(G_{0} .3\right)$, we have in particular that

$$
F_{1}\left(t, u_{t}^{n}\right) \rightarrow F_{1}\left(t, u_{t}\right) \text { in } I^{2}\left(0, T ; V^{*}\right),
$$

and

$$
G_{0}\left(t, u_{t}^{n}\right) \rightarrow G_{0}\left(t, u_{t}\right) \text { in } I^{2}(0, T ; H) .
$$

Moreover, by $(A .3)$, the sequence $\left\{A\left(t, u^{n}(t)\right)\right\}_{n \geq 1}$ is bounded in $I^{2}\left(0, T ; V^{*}\right)$. Thus, there exist a subsequence $\left\{A\left(t, u^{n_{k}}(t)\right)\right\}_{n_{k} \geq 1} \subset\left\{A\left(t, u^{n}(t)\right)\right\}_{n \geq 1}$ and $\xi \in I^{2}\left(0, T ; V^{*}\right)$, such that

$$
A\left(t, u^{n_{k}}(t)\right) \rightarrow \xi \text { in } I^{2}\left(0, T ; V^{*}\right),
$$

where $\rightarrow$ denotes weak convergence. Thus, we can take limits in $\left(P_{n_{k}}^{\prime}\right)$, and obtain that $u$ is solution of

$$
\left\{\begin{aligned}
u \in I^{2}( & -h, T ; V) \cap L^{2}(\Omega ; C(-h, T ; H)), \\
u(t)= & \psi(0)+\int_{0}^{t} \xi(s) d s+\int_{0}^{t}\left(F_{1}\left(s, u_{s}\right)+f(s)\right) d s \\
& \quad+\int_{0}^{t}\left(G_{0}\left(s, u_{s}\right)+g(s)\right) d W(s), t \in[0, T], \\
u(t)= & \psi(t), t \in[-h, 0] .
\end{aligned}\right.
$$

To simplify the notation, observe that $\xi$ is uniquely determined by $u$, and thus, the whole sequence $\left\{A\left(t, u^{n}(t)\right)\right\}_{n \geq 1}$ converges weakly to $\xi$ in $I^{2}\left(0, T ; V^{*}\right)$.

In order to prove that $u$ is in fact a solution of problem $\left(P^{\prime}\right)$, we only need to prove that $\xi(t)=A(t, u(t))$ in $(0, T)$.

First of all, applying Itô's formula to $\left|u^{n}(t)\right|^{2}$ and to $|u(t)|^{2}$ on the interval $[0, T]$, we obtain

$$
\begin{aligned}
E\left|u^{n}(T)\right|^{2}= & E|\psi(0)|^{2}+2 E \int_{0}^{T}\left\langle A\left(s, u^{n}(s)\right), u^{n}(s)\right\rangle d s \\
& +\lambda E \int_{0}^{T}\left(u^{n}(s), u^{n-1}(s)\right) d s \\
& -\lambda E \int_{0}^{T}\left|u^{n}(s)\right|^{2} d s+2 E \int_{0}^{T}\left\langle F_{1}\left(s, u_{s}^{n-1}\right)+f(s), u^{n}(s)\right\rangle d s \\
& +E \int_{0}^{T}\left|G_{0}\left(s, u_{s}^{n-1}\right)+g(s)\right|^{2} d s
\end{aligned}
$$

and

$$
\begin{aligned}
E|u(T)|^{2}=E|\psi(0)|^{2} & +2 E \int_{0}^{T}\langle\xi(s), u(s)\rangle d s+2 E \int_{0}^{T}\left\langle F_{1}\left(s, u_{s}\right)+f(s), u(s)\right\rangle d s \\
& +E \int_{0}^{T}\left|G_{0}\left(s, u_{s}\right)+g(s)\right|^{2} d s
\end{aligned}
$$


Taking limits in (2.12) as $n \rightarrow \infty$, and comparing with (2.13), we deduce:

$$
\lim _{n \rightarrow \infty} E \int_{0}^{T}\left\langle A\left(s, u^{n}(s)\right), u^{n}(s)\right\rangle d s=E \int_{0}^{T}\langle\xi(s), u(s)\rangle d s .
$$

In particular, thanks to $(A .4)$,

$$
-2 E \int_{0}^{T}\left\langle A\left(s, u^{n}(s)\right)-A(s, X(s)), u^{n}(s)-X(s)\right\rangle d s+\lambda E \int_{0}^{T}\left|u^{n}(s)-X(s)\right|^{2} d s \geq 0,
$$

for all $X \in I^{2}(0, T ; V)$ and all $n \geq 1$. Taking limits in (2.15), we get

$$
-2 E \int_{0}^{T}\langle\xi(s)-A(s, X(s)), u(s)-X(s)\rangle d s+\lambda E \int_{0}^{T}|u(s)-X(s)|^{2} d s \geq 0,
$$

for all $X \in I^{2}(0, T ; V)$. Now, if we set $X=u-\delta Z$, with $Z \in I^{2}(0, T ; V)$ and $\delta>0$, we have

$$
-2 E \int_{0}^{T}\langle\xi(s)-A(s, u(s)-\delta Z(s)), \delta Z(s)\rangle d s+\lambda \delta^{2} E \int_{0}^{T}|Z(s)|^{2} d s \geq 0,
$$

for all $Z \in I^{2}(0, T ; V)$ and all $\delta>0$.

If we divide by $\delta$ in (2.16) and take limits as $\delta \rightarrow 0$, we obtain from $(A .2)$

$$
E \int_{0}^{T}\langle\xi(s)-A(s, u(s)), Z(s)\rangle d s \leq 0, \forall Z \in I^{2}(0, T ; V),
$$

and, therefore, $A(s, u(s))=\xi(s)$ in $(0, T)$.

Remark 2.1. We want to point out once again that in [4], a similar result to the preceding theorem is obtained but under the assumption of being $F_{1}(t, \cdot)$ a family of operators taking values in $H$ instead of $V^{*}$.

\section{Existence and uniqueness of solution of problem $(P)$}

Now, under suitable additional assumptions, we can show existence and uniqueness of solution of problem $(P)$. In fact, we will prove the following theorem:

Theorem 3.1. Assume that the injection $V \subset H$ is compact, and hypotheses (A.1)$(A .4),\left(F_{1} .1\right)-\left(F_{1} .3\right),\left(F_{2} .1\right)-\left(F_{2} .4\right),\left(G_{0} .1\right)-\left(G_{0} .3\right)$ and $\left(G_{1} .1\right)-\left(G_{1} .4\right)$ hold. Suppose also the three following hypotheses:

(A.5) There exists $\widehat{\lambda}>0$ such that for all $x, y \in L^{2}(-h, T ; V)$ and for all $t \in[0, T]$ it holds

$$
\begin{aligned}
\alpha \int_{0}^{t}\|x(s)-y(s)\|^{2} d s+\int_{0}^{t}\left|G_{1}\left(s, x_{s}\right)-G_{1}\left(s, y_{s}\right)\right|^{2} d s \\
\leq-2 \int_{0}^{t}\langle A(s, x(s))-A(s, y(s)), x(s)-y(s)\rangle d s+\lambda \int_{0}^{t}|x(s)-y(s)|^{2} d s \\
\quad+\hat{\lambda} \int_{-h}^{0}\|x(s)-y(s)\|^{2} d s-2 \int_{0}^{t}\left\langle F_{2}\left(s, x_{s}\right)-F_{2}\left(s, y_{s}\right), x(s)-y(s)\right\rangle d s .
\end{aligned}
$$


$\left(F_{1} .4\right)$ There exists $C^{F_{1}}>0$ such that for all $X, Y \in L^{2}(\Omega ; C(-h, T ; H))$ such that $X \equiv Y$ on $[-h, 0]$, and all $t \in[0, T]$,

$$
E \int_{0}^{t}\left\|F_{1}\left(s, X_{s}\right)-F_{1}\left(s, Y_{s}\right)\right\|_{*}^{2} d s \leq C^{F_{1}} \int_{0}^{t} \sup _{0 \leq \theta \leq s} E|X(\theta)-Y(\theta)|^{2} d s .
$$

$\left(G_{0} .4\right)$ There exists $C^{G_{0}}>0$ such that for all $X, Y \in L^{2}(\Omega ; C(-h, T ; H))$ such that $X \equiv Y$ on $[-h, 0]$, and all $t \in[0, T]$,

$$
E \int_{0}^{t}\left|G_{0}\left(s, X_{s}\right)-G_{0}\left(s, Y_{s}\right)\right|^{2} d s \leq C^{G_{0}} \int_{0}^{t} \sup _{0 \leq \theta \leq s} E|X(\theta)-Y(\theta)|^{2} d s .
$$

Then, for every $\psi \in I^{2}(-h, 0 ; V) \cap L^{2}(\Omega ; C(-h, 0 ; H)), f \in I^{2}\left(0, T ; V^{*}\right)$ and $g \in I^{2}(0, T ; H)$, there exists a unique solution $u$ of the problem $(P)$.

\section{Proof.}

Uniqueness of solutions. Assume that $u, v \in I^{2}(-h, T ; V) \cap L^{2}(\Omega ; C(-h, T ; H))$ are two solutions to problem $(P)$. Then, applying Itô's formula to $|u(t)-v(t)|^{2}$, and using $(A .5)$, we obtain for each $t \in[0, T]$

$$
\begin{aligned}
& |u(t)-v(t)|^{2}+\alpha \int_{0}^{t}\|u(s)-v(s)\|^{2} d s \\
& \leq \lambda \int_{0}^{t}|u(s)-v(s)|^{2} d s+2 \int_{0}^{t}\left\langle F_{1}\left(s, u_{s}\right)-F_{1}\left(s, v_{s}\right), u(s)-v(s)\right\rangle d s \\
& \quad+2 \int_{0}^{t}\left(G_{0}\left(s, u_{s}\right)-G_{0}\left(s, v_{s}\right), G_{1}\left(s, u_{s}\right)-G_{1}\left(s, v_{s}\right)\right) d s \\
& \quad+2 \int_{0}^{t}\left(G_{0}\left(s, u_{s}\right)-G_{0}\left(s, v_{s}\right), u(s)-v(s)\right) d W(s) \\
& \quad+2 \int_{0}^{t}\left(G_{1}\left(s, u_{s}\right)-G_{1}\left(s, v_{s}\right), u(s)-v(s)\right) d W(s) \\
& \quad+\int_{0}^{t}\left|G_{0}\left(s, u_{s}\right)-G_{0}\left(s, v_{s}\right)\right|^{2} d s .
\end{aligned}
$$

Therefore,

$$
\begin{aligned}
& E|u(t)-v(t)|^{2}+\alpha E \int_{0}^{t}\|u(s)-v(s)\|^{2} d s \\
& \leq \lambda E \int_{0}^{t}|u(s)-v(s)|^{2} d s+E \int_{0}^{t}\left|G_{0}\left(s, u_{s}\right)-G_{0}\left(s, v_{s}\right)\right|^{2} d s \\
&+2 E \int_{0}^{t}\left(G_{0}\left(s, u_{s}\right)-G_{0}\left(s, v_{s}\right), G_{1}\left(s, u_{s}\right)-G_{1}\left(s, v_{s}\right)\right) d s \\
&+2 E \int_{0}^{t}\left\langle F_{1}\left(s, u_{s}\right)-F_{1}\left(s, v_{s}\right), u(s)-v(s)\right\rangle d s,
\end{aligned}
$$

for all $t \in[0, T]$. We can now deduce from $\left(F_{1} .4\right)$ that

$$
\begin{aligned}
2 E \int_{0}^{t}\left\langle F_{1}\left(s, u_{s}\right)-F_{1}\left(s, v_{s}\right), u(s)-v(s)\right\rangle d s \leq & \frac{3}{\alpha} C^{F_{1}} \int_{0}^{t} \sup _{0 \leq \theta \leq s} E|u(\theta)-v(\theta)|^{2} d s \\
& +\frac{\alpha}{3} \int_{0}^{t} E\|u(s)-v(s)\|^{2} d s .
\end{aligned}
$$


From $\left(G_{1} .4\right)$ it follows that

$$
\begin{aligned}
2 E & \int_{0}^{t}\left(G_{0}\left(s, u_{s}\right)-G_{0}\left(s, v_{s}\right), G_{1}\left(s, u_{s}\right)-G_{1}\left(s, v_{s}\right)\right) d s \\
& \leq 2 E \int_{0}^{t}\left|G_{0}\left(s, u_{s}\right)-G_{0}\left(s, v_{s}\right)\right|\left|G_{1}\left(s, u_{s}\right)-G_{1}\left(s, v_{s}\right)\right| d s \\
& \leq \frac{3 K_{G_{1}}}{\alpha} E \int_{0}^{t}\left|G_{0}\left(s, u_{s}\right)-G_{0}\left(s, v_{s}\right)\right|^{2} d s+\frac{\alpha}{3} E \int_{0}^{t}\|u(s)-v(s)\|^{2} d s .
\end{aligned}
$$

Thanks to condition $\left(G_{0} .4\right)$, we have

$$
\begin{aligned}
\left(\frac{3 K_{G_{1}}}{\alpha}+1\right) & E \int_{0}^{t}\left|G_{0}\left(s, u_{s}\right)-G_{0}\left(s, v_{s}\right)\right|^{2} d s \\
& \leq\left(\frac{3 K_{G_{1}}}{\alpha}+1\right) C^{G_{0}} \int_{0}^{t} \sup _{0 \leq \theta \leq s} E|u(\theta)-v(\theta)|^{2} d s .
\end{aligned}
$$

From (3.1)-(3.4), it follows that for all $t \in[0, T]$

$$
\begin{aligned}
& \sup _{0 \leq s \leq t} E|u(s)-v(s)|^{2}+\frac{\alpha}{3} E \int_{0}^{t}\|u(s)-v(s)\|^{2} d s \\
& \quad \leq 2\left(|\lambda|+\left(\frac{3 K_{G_{1}}}{\alpha}+1\right) C^{G_{0}}+\frac{3}{\alpha} C^{F_{1}}\right) \int_{0}^{t} \sup _{0 \leq \theta \leq s} E|u(\theta)-v(\theta)|^{2} d s,
\end{aligned}
$$

and Gronwall's lemma implies now uniqueness.

Existence of solutions. We will proceed in two steps.

Step 1. Firstly, we consider that $F_{1} \equiv G_{0} \equiv 0$ and $\lambda=0$. We have to prove existence of solution to

$$
\left\{\begin{aligned}
u \in & I^{2}(-h, T ; V) \cap L^{2}(\Omega ; C(-h, T ; H)), \\
u(t) & =\psi(0)+\int_{0}^{t} A(s, u(s)) d s+\int_{0}^{t}\left(F_{2}\left(s, u_{s}\right)+f(s)\right) d s \\
& +\int_{0}^{t}\left(G_{1}\left(s, u_{s}\right)+g(s)\right) d W(s), t \in[0, T], \\
u(t) & =\psi(t), t \in[-h, 0] .
\end{aligned}\right.
$$

We will use a Galerkin scheme. As injection $V \subset H$ is compact, there exists a Hilbert basis of $H,\left\{v_{i}\right\}_{i \geq 1} \subset V$ and a nondecreasing sequence $\left\{\mu_{i}\right\}_{i \geq 1}$ of positive numbers, with $\lim _{i \rightarrow \infty} \mu_{i}=+\infty$, such that $\left(\left(w, v_{i}\right)\right)=\mu_{i}\left(w, v_{i}\right) \forall i \geq 1, \forall w \in V$. The subspace of $V$ spanned by $v_{1}, \ldots, v_{m}$ will be denoted by $V_{m}$. Consider the projector $P_{m}: H \rightarrow V_{m}$ given by $P_{m} u=\sum_{i=1}^{m}\left(u, v_{i}\right) v_{i}$, and define $u^{m}(t)=\sum_{i=1}^{m} \gamma^{m i}(t) v_{i}$, where

$$
\left\{\begin{array}{l}
u^{m} \in I^{2}\left(-h, T ; V_{m}\right) \cap L^{2}\left(\Omega ; C\left(-h, T ; V_{m}\right)\right), \\
\left(u^{m}(t), v\right)=\left(P_{m} \psi(0), v\right)+\int_{0}^{t}\left\langle A\left(s, u^{m}(s)\right)+F_{2}\left(s, u_{s}^{m}\right)+f(s), v\right\rangle d s \\
\quad+\int_{0}^{t}\left(G_{1}\left(s, u_{s}^{m}\right)+g(s), v\right) d W(s), \forall t \in[0, T], \forall v \in V_{m}, \\
u^{m}(t)=P_{m} \psi(t), t \in[-h, 0] .
\end{array}\right.
$$


The existence and uniqueness of solution to problem $\left(\widehat{P}^{m}\right)$ is therefore guaranteed by Theorem 2.1 (notice that in this case, $V=H=V^{*}=V_{m}$ ).

Observe also that $u^{m}=P_{m} \psi$ in $[-h, 0]$, and, by the particular choice of the basis $\left\{v_{i}\right\}$, for each $m \geq 1,\left\|u^{m}(t)\right\| \leq\|\psi(t)\|$ for all $t \in[-h, 0]$, and the sequence $u^{m}$ converges to $\psi$ in $I^{2}(-h, 0 ; V) \cap L^{2}(\Omega ; C(-h, 0 ; H))$.

Applying Itô's formula to $\left|u^{m}(t)\right|^{2}$, and using $A(t, 0)=0$ a.e.t $\in(0, T),(A .5),\left(F_{2} .2\right)$ and $\left(G_{1} .2\right)$, we have (bearing in mind that we are assuming $\lambda=0$ ):

$$
\begin{aligned}
\left|u^{m}(t)\right|^{2}+\alpha \int_{0}^{t}\left\|u^{m}(s)\right\|^{2} d s & \leq\left|P_{m} \psi(0)\right|^{2}+2 \int_{0}^{t}\left\langle f(s), u^{m}(s)\right\rangle d s+\int_{0}^{t}|g(s)|^{2} d s \\
& +2 \int_{0}^{t}\left(G_{1}\left(s, u_{s}^{m}\right), g(s)\right) d s+\widehat{\lambda} \int_{-h}^{0}\left\|P_{m} \psi(s)\right\|^{2} d s \\
& +2 \int_{0}^{t}\left(G_{1}\left(s, u_{s}^{m}\right)+g(s), u^{m}(s)\right) d W(s)
\end{aligned}
$$

for all $t \in[0, T]$, and therefore,

$$
\begin{aligned}
E\left|u^{m}(t)\right|^{2}+\alpha E \int_{0}^{t}\left\|u^{m}(s)\right\|^{2} d s & \leq E|\psi(0)|^{2}+2 E \int_{0}^{t}\left\langle f(s), u^{m}(s)\right\rangle d s+E \int_{0}^{t}|g(s)|^{2} d s \\
& +\widehat{\lambda} E \int_{-h}^{0}\|\psi(s)\|^{2} d s+2 E \int_{0}^{t}\left(G_{1}\left(s, u_{s}^{m}\right), g(s)\right) d s,
\end{aligned}
$$

for all $t \in[0, T]$. But

$$
2 E \int_{0}^{t}\left\langle f(s), u^{m}(s)\right\rangle d s \leq \frac{3}{\alpha} E \int_{0}^{t}\|f(s)\|_{*}^{2} d s+\frac{\alpha}{3} E \int_{0}^{t}\left\|u^{m}(s)\right\|^{2} d s,
$$

and, by $\left(G_{1} .2\right)$ and $\left(G_{1} .4\right)$,

$$
2 E \int_{0}^{t}\left(G_{1}\left(s, u_{s}^{m}\right), g(s)\right) d s \leq \frac{3 K_{G_{1}}}{\alpha} E \int_{0}^{t}|g(s)|^{2} d s+\frac{\alpha}{3} E \int_{-h}^{t}\left\|u^{m}(s)\right\|^{2} d s .
$$

Then, from (3.5) we can obtain

$$
\begin{aligned}
E\left|u^{m}(t)\right|^{2}+\frac{\alpha}{3} E \int_{0}^{t}\left\|u^{m}(s)\right\|^{2} d s & \leq E|\psi(0)|^{2}+\frac{3}{\alpha} E \int_{0}^{t}\|f(s)\|_{*}^{2} d s \\
& +\left(\widehat{\lambda}+\frac{\alpha}{3}\right) E \int_{-h}^{0}\|\psi(s)\|^{2} d s \\
& +\left(1+\frac{3 K_{G_{1}}}{\alpha}\right) E \int_{0}^{t}|g(s)|^{2} d s
\end{aligned}
$$

for all $t \in[0, T]$, and, consequently, the sequence $\left\{u^{m}\right\}_{m \geq 1}$ is bounded in $I^{2}(-h, T ; V)$ (notice that $u^{m}$ converges to $\psi$ in $\left.I^{2}(-h, 0 ; V) \cap L^{2}(\Omega ; C(-h, 0 ; H))\right)$, and the sequence $\left\{u^{m}(T)\right\}_{m \geq 1}$ is bounded in $L^{2}(\Omega ; H)$. In fact, from $(A .3),\left(F_{2} .2\right),\left(F_{2} .4\right),\left(G_{1} .2\right)$ and $\left(G_{1} .4\right)$, we can affirm that

$$
\begin{gathered}
\left\{u^{m}(\cdot)\right\}_{m \geq 1} \text { is bounded in } I^{2}(-h, T ; V), \\
\left\{u^{m}(T)\right\}_{m \geq 1} \text { is bounded in } L^{2}(\Omega ; H),
\end{gathered}
$$




$$
\begin{gathered}
\left\{A\left(\cdot, u^{m}(\cdot)\right)\right\}_{m \geq 1} \text { is bounded in } I^{2}\left(0, T ; V^{*}\right), \\
\left\{F_{2}\left(\cdot, u_{\cdot}^{m}\right)\right\}_{m \geq 1} \text { is bounded in } I^{2}\left(0, T ; V^{*}\right), \\
\left\{G_{1}\left(\cdot, u^{m}\right)\right\}_{m \geq 1} \text { is bounded in } I^{2}(0, T ; H) .
\end{gathered}
$$

Thus, we can ensure that there exists a subsequence $\left\{u^{m_{k}}\right\}$ of $\left\{u^{m}\right\}$, a random variable $\xi \in$ $L^{2}(\Omega ; H)$ and four processes $u \in I^{2}(-h, T ; V), \eta \in I^{2}\left(0, T ; V^{*}\right), \sigma \in I^{2}\left(0, T ; V^{*}\right)$, and $\zeta \in$ $I^{2}(0, T ; H)$ such that

$$
\begin{gathered}
u^{m_{k}}(T) \rightarrow \xi \text { in } L^{2}(\Omega ; H), \\
u^{m_{k}}(\cdot) \rightarrow u \text { in } I^{2}(-h, T ; V), \\
A\left(\cdot, u^{m_{k}}(\cdot)\right) \rightarrow \eta \text { in } I^{2}\left(0, T ; V^{*}\right), \\
F_{2}\left(\cdot, u_{\cdot}^{m_{k}}\right) \rightarrow \sigma \text { in } I^{2}\left(0, T ; V^{*}\right), \\
G_{1}\left(\cdot, u_{\cdot}^{m_{k}}\right) \rightarrow \zeta \text { in } I^{2}(0, T ; H) .
\end{gathered}
$$

Now, let $\chi$ be an absolutely continuous real function on $[0, T]$ such that $\chi^{\prime} \in L^{2}(0, T)$ and $\chi(T)=0$. Fix $m_{j}$ and $v \in V_{m_{j}}$. Applying Itô's formula to $\left(u^{m_{k}}(t), v\right) \chi(t)$ with $1 \leq m_{j} \leq m_{k}$, we get

$$
\begin{aligned}
0= & \left(P_{m_{k}} \psi(0), v\right) \chi(0)+\int_{0}^{T}\left\langle A\left(s, u^{m_{k}}(s)\right)+F_{2}\left(s, u_{s}^{m_{k}}\right)+f(s), v\right\rangle \chi(s) d s \\
& +\int_{0}^{T}\left(G_{1}\left(s, u_{s}^{m_{k}}\right)+g(s), v\right) \chi(s) d W(s)+\int_{0}^{T}\left(u^{m_{k}}(s), v\right) \chi^{\prime}(s) d s .
\end{aligned}
$$

We can take limits in (3.6) as $m_{k} \rightarrow \infty$, and observing that $m_{j}$ is arbitrary and that $\cup_{m \geq 1} V_{m}$ is dense in $V$, we can ensure that

$$
\begin{aligned}
0= & (\psi(0), v) \chi(0)+\int_{0}^{T}\langle\eta(s)+\sigma(s)+f(s), v\rangle \chi(s) d s \\
& +\int_{0}^{T}(\zeta(s)+g(s), v) \chi(s) d W(s)+\int_{0}^{T}(u(s), v) \chi^{\prime}(s) d s, \forall v \in V .
\end{aligned}
$$

Consequently, if we fix $t \in(0, T)$, and for each integer $n \geq 1$ such that $t+1 / 2 n \leq T$ we denote by $\chi^{n}$ the function

$$
\chi^{n}(s)= \begin{cases}1 & \text { if } 0 \leq s \leq t-1 / 2 n, \\ \frac{1}{2}+n(t-s) & \text { if } t-1 / 2 n \leq s \leq t+1 / 2 n, \\ 0 & \text { if } t+1 / 2 n \leq s \leq T,\end{cases}
$$

we deduce from (3.7),

$$
\begin{aligned}
n \int_{t-1 / 2 n}^{t+1 / 2 n}(u(s), v) d s= & (\psi(0), v)+\int_{0}^{T}\langle\eta(s)+\sigma(s)+f(s), v\rangle \chi^{n}(s) d s \\
& +\int_{0}^{T}(\zeta(s)+g(s), v) \chi^{n}(s) d W(s), \forall v \in V .
\end{aligned}
$$


We can take limits in (3.8) a.e. $t \in(0, T)$, and obtain

$$
(u(t), v)=(\psi(0), v)+\int_{0}^{t}\langle\eta(s)+\sigma(s)+f(s), v\rangle d s+\int_{0}^{t}(\zeta(s)+g(s), v) d W(s),
$$

a.e. $t \in(0, T)$, for all $v \in V$. Taking into account the separability of $V,(3.9)$ implies

$$
u(t)=\psi(0)+\int_{0}^{t}(\eta(s)+\sigma(s)+f(s)) d s+\int_{0}^{t}(\zeta(s)+g(s)) d W(s) \text { in } V^{*},
$$

a.e. $t \in(0, T)$.

Thus, $u$ is a.e. in $[0, T]$ equal to a process in $L^{2}(\Omega ; C(0, T ; H))$ which satisfies $(3.10)$ for all $t \in[0, T]$. We denote again by $u$ such a process, and thus $u$ satisfies

$$
\left\{\begin{array}{l}
u \in I^{2}(-h, T ; V) \cap L^{2}(\Omega ; C(-h, T ; H)) \\
u(t)=\psi(0)+\int_{0}^{t}(\eta(s)+\sigma(s)+f(s)) d s+\int_{0}^{t}(\zeta(s)+g(s)) d W(s), t \in[0, T], \\
u(T)=\xi \\
u(t)=\psi(t), t \in[-h, 0] .
\end{array}\right.
$$

Now, to finish the proof, it is enough to prove that $\eta(s)+\sigma(s)=A(s, u(s))+F_{2}\left(s, u_{s}\right)$ and $\zeta(s)=G_{1}\left(s, u_{s}\right)$.

Consider $X \in I^{2}(-h, T ; V)$ such that $X=\psi$ in $(-h, 0)$, and denote

$$
\begin{aligned}
x^{m_{k}} & =2 E \int_{0}^{T}\left\langle A\left(t, u^{m_{k}}(t)\right)-A(t, X(t))+F_{2}\left(t, u_{t}^{m_{k}}\right)-F_{2}\left(t, X_{t}\right), u^{m_{k}}(t)-X(t)\right\rangle d t \\
& +\alpha E \int_{0}^{T}\left\|u^{m_{k}}(t)-X(t)\right\|^{2} d t-\widehat{\lambda} E \int_{-h}^{0}\left\|\psi(t)-P_{m_{k}} \psi(t)\right\|^{2} d s \\
& +E \int_{0}^{T}\left|G_{1}\left(t, u_{t}^{m_{k}}\right)-G_{1}\left(t, X_{t}\right)\right|^{2} d t,
\end{aligned}
$$

$y^{m_{k}}=2 E \int_{0}^{T}\left\langle A\left(t, u^{m_{k}}(t)\right)+F_{2}\left(t, u_{t}^{m_{k}}\right), u^{m_{k}}(t)\right\rangle d t+E \int_{0}^{T}\left|G_{1}\left(t, u_{t}^{m_{k}}\right)\right|^{2} d t+\alpha E \int_{0}^{T}\left\|u^{m_{k}}(t)\right\|^{2} d t$.

Then, thanks to condition $(A .5)$ (with $\lambda=0$ ), $x^{m_{k}} \leq 0$.

Observe also that

$$
\begin{aligned}
x^{m_{k}}-y^{m_{k}} & =2 E \int_{0}^{T}\left\langle-A(t, X(t))-F_{2}\left(t, X_{t}\right), u^{m_{k}}(t)\right\rangle d t \\
& +2 E \int_{0}^{T}\left\langle A\left(t, u^{m_{k}}(t)\right)-A(t, X(t))+F_{2}\left(t, u_{t}^{m_{k}}\right)-F_{2}\left(t, X_{t}\right),-X(t)\right\rangle d t \\
& +E \int_{0}^{T}\left|G_{1}\left(t, X_{t}\right)\right|^{2} d t-2 E \int_{0}^{T}\left(G_{1}\left(t, u_{t}^{m_{k}}\right), G_{1}\left(t, X_{t}\right)\right) d t+\alpha E \int_{0}^{T}\|X(t)\|^{2} d t \\
& -2 \alpha E \int_{0}^{T}\left(\left(u^{m_{k}}(t), X(t)\right)\right) d t-\widehat{\lambda} E \int_{-h}^{0}\left\|\psi(t)-P_{m_{k}} \psi(t)\right\|^{2} d s,
\end{aligned}
$$


and consequently,

$$
\begin{aligned}
\lim _{k \rightarrow \infty}\left(x^{m_{k}}-y^{m_{k}}\right)= & 2 E \int_{0}^{T}\left\langle-A(t, X(t))-F_{2}\left(t, X_{t}\right), u(t)\right\rangle d t \\
& +2 E \int_{0}^{T}\left\langle\eta(t)-A(t, X(t))+\sigma(t)-F_{2}\left(t, X_{t}\right),-X(t)\right\rangle d t \\
& +E \int_{0}^{T}\left|G_{1}\left(t, X_{t}\right)\right|^{2} d t-2 E \int_{0}^{T}\left(\zeta(t), G_{1}\left(t, X_{t}\right)\right) d t \\
& -2 \alpha E \int_{0}^{T}((u(t), X(t))) d t+\alpha E \int_{0}^{T}\|X(t)\|^{2} d t .
\end{aligned}
$$

Applying Itô's formula to $\left|u^{m_{k}}(t)\right|^{2}$ on the interval $[0, T]$,

$$
\begin{aligned}
E\left|u^{m_{k}}(T)\right|^{2} \leq & E|\psi(0)|^{2}+E \int_{0}^{T}\left|G_{1}\left(t, u_{t}^{m_{k}}\right)+g(t)\right|^{2} d t \\
& +2 E \int_{0}^{T}\left\langle A\left(t, u^{m_{k}}(t)\right)+F_{2}\left(t, u_{t}^{m_{k}}\right)+f(t), u^{m_{k}}(t)\right\rangle d t
\end{aligned}
$$

and, thus

$$
\begin{gathered}
y^{m_{k}} \geq E\left|u^{m_{k}}(T)\right|^{2}-E|\psi(0)|^{2}-E \int_{0}^{T}|g(t)|^{2} d t+\alpha E \int_{0}^{T}\left\|u^{m_{k}}(t)\right\|^{2} d t \\
-2 E \int_{0}^{T}\left(G_{1}\left(t, u_{t}^{m_{k}}\right), g(t)\right) d t-2 E \int_{0}^{T}\left\langle f(t), u^{m_{k}}(t)\right\rangle d t .
\end{gathered}
$$

Letting $k \rightarrow \infty$,

$$
\begin{aligned}
\liminf _{k \rightarrow \infty} y^{m_{k}} \geq & E|u(T)|^{2}-E|\psi(0)|^{2}-E \int_{0}^{T}|g(t)|^{2} d t+\alpha E \int_{0}^{T}\|u(t)\|^{2} d t \\
& -2 E \int_{0}^{T}(\zeta(t), g(t)) d t-2 E \int_{0}^{T}\langle f(t), u(t)\rangle d t .
\end{aligned}
$$

Applying once again Itô's formula to $|u(t)|^{2}$ on $[0, T]$,

$$
E|u(T)|^{2}=E|\psi(0)|^{2}+E \int_{0}^{T}|\zeta(t)+g(t)|^{2} d t+2 E \int_{0}^{T}\langle\eta(t)+\sigma(t)+f(t), u(t)\rangle d t,
$$

and so, from (3.13)

$$
\liminf _{k \rightarrow \infty} y^{m_{k}} \geq 2 E \int_{0}^{T}\langle\eta(t)+\sigma(t), u(t)\rangle d t+E \int_{0}^{T}|\zeta(t)|^{2} d t+\alpha E \int_{0}^{T}\|u(t)\|^{2} d t .
$$

From (3.12) and (3.14) we have

$$
\begin{gathered}
0 \geq \liminf _{k \rightarrow \infty} x^{m_{k}} \geq 2 E \int_{0}^{T}\left\langle\eta(t)-A(t, X(t))+\sigma(t)-F_{2}\left(t, X_{t}\right), u(t)-X(t)\right\rangle d t \\
+E \int_{0}^{T}\left|\zeta(t)-G_{1}\left(t, X_{t}\right)\right|^{2} d t+\alpha E \int_{0}^{T}\|u(t)-X(t)\|^{2} d t
\end{gathered}
$$


If we take $X(t)=u(t)$ in (3.15), it follows that $\zeta(t)=G_{1}\left(t, u_{t}\right), t \in[0, T]$. Now, we will set $X(t)=u(t)-\delta Z(t)$, where $\delta>0$ and $Z \in I^{2}(-h, T ; V)$ is such that $Z=0$ in $(-h, 0)$. Then, by $(3.15)$,

$$
\begin{gathered}
0 \geq 2 E \int_{0}^{T}\left\langle\eta(t)-A(t, u(t)-\delta Z(t))+\sigma(t)-F_{2}\left(t, u_{t}-\delta Z_{t}\right), \delta Z(t)\right\rangle d t \\
+E \int_{0}^{T}\left|G_{1}\left(t, u_{t}\right)-G_{1}\left(t, u_{t}-\delta Z_{t}\right)\right|^{2} d t .
\end{gathered}
$$

Dividing by $\delta$ in (3.16), and letting $\delta \rightarrow 0$, we get by $(A .2),\left(F_{2} .2\right)$ and $\left(F_{2} .4\right)$,

$$
2 E \int_{0}^{T}\left\langle\eta(t)-A(t, u(t))+\sigma(t)-F_{2}\left(t, u_{t}\right), Z(t)\right\rangle d t \leq 0,
$$

and since $Z \in I^{2}(0, T ; V)$ is arbitrary, clearly $\eta(t)+\sigma(t)=A(t, u(t))+F_{2}\left(t, u_{t}\right)$ in $[0, T]$.

Step 2. Now, we consider problem $(P)$ under the conditions in the theorem. We denote $u^{0} \equiv 0$, and define by recurrence a sequence $\left\{u^{n}\right\}_{n \geq 1}$ of processes by

$$
\left\{\begin{aligned}
u^{n} \in & I^{2}(-h, T ; V) \cap L^{2}(\Omega ; C(-h, T ; H)), \\
u^{n}(t) & =\psi(0)+\int_{0}^{t}\left(A\left(s, u^{n}(s)\right)-\frac{\lambda}{2} u^{n}(s)\right) d s+\frac{\lambda}{2} \int_{0}^{t} u^{n-1}(s) d s \\
& +\int_{0}^{t}\left(F_{1}\left(s, u_{s}^{n-1}\right)+F_{2}\left(s, u_{s}^{n}\right)+f(s)\right) d s \\
& +\int_{0}^{t}\left(G_{0}\left(s, u_{s}^{n-1}\right)+G_{1}\left(s, u_{s}^{n}\right)+g(s)\right) d W(s), t \in[0, T], \\
u^{n}(t) & =\psi(t), t \in[-h, 0] .
\end{aligned}\right.
$$

Observe that if $u^{n-1} \in I^{2}(-h, T ; V) \cap L^{2}(\Omega ; C(-h, T ; H))$, then $F_{1}\left(t, u_{t}^{n-1}\right) \in I^{2}\left(0, T ; V^{*}\right)$, and $G_{0}\left(t, u_{t}^{n-1}\right) \in I^{2}(0, T ; H)$. Moreover, the family of operators defined by $\widetilde{A}(t, v)=A(t, v)-$ $\frac{\lambda}{2} v \forall v \in V$, a.e.t $\in(0, T)$, satisfies conditions $(A .1)-(A .5)$ with $\lambda=0$. Consequently, we can use Step 1 to ensure that problem $\left(P^{n}\right)$ has a unique solution.

Now, arguing as in the proof of Theorem 2.1, we can prove that $\left\{u^{n}\right\}_{n \geq 1}$ is a Cauchy sequence in $I^{2}(-h, T ; V) \cap L^{2}(\Omega ; C(-h, T ; H))$, and thus, it converges to a process $u \in$ $I^{2}(-h, T ; V) \cap L^{2}(\Omega ; C(-h, T ; H))$, which will be the solution to $(P)$.

In order to obtain our objective, we first apply Itô's formula to the process $u^{n+1}(t)-u^{n}(t)$, $t \geq 0, n \geq 1$, and using (A.5) we have

$$
\begin{aligned}
& \left|u^{n+1}(t)-u^{n}(t)\right|^{2}+\alpha \int_{0}^{t}\left\|u^{n+1}(s)-u^{n}(s)\right\|^{2} d s \\
& \quad \leq \lambda \int_{0}^{t}\left(u^{n+1}(s)-u^{n}(s), u^{n}(s)-u^{n-1}(s)\right) d s \\
& \quad+2 \int_{0}^{t}\left\langle F_{1}\left(s, u_{s}^{n}\right)-F_{1}\left(s, u_{s}^{n-1}\right), u^{n+1}(s)-u^{n}(s)\right\rangle d s \\
& \quad+2 \int_{0}^{t}\left(G_{0}\left(s, u_{s}^{n}\right)-G_{0}\left(s, u_{s}^{n-1}\right), G_{1}\left(s, u_{s}^{n+1}\right)-G_{1}\left(s, u_{s}^{n}\right) d s\right.
\end{aligned}
$$




$$
\begin{aligned}
& +\int_{0}^{t}\left|G_{0}\left(s, u_{s}^{n}\right)-G_{0}\left(s, u_{s}^{n-1}\right)\right|^{2} d s \\
& +2 \int_{0}^{t}\left(G_{0}\left(s, u_{s}^{n}\right)-G_{0}\left(s, u_{s}^{n-1}\right), u^{n+1}(s)-u^{n}(s)\right) d W(s) \\
& +2 \int_{0}^{t}\left(G_{1}\left(s, u_{s}^{n+1}\right)-G_{1}\left(s, u_{s}^{n}\right), u^{n+1}(s)-u^{n}(s)\right) d W(s),
\end{aligned}
$$

which, together with conditions $\left(F_{1} .4\right),\left(G_{0} .4\right)$ and $\left(G_{1} .4\right)$ imply

$$
\begin{aligned}
& E\left|u^{n+1}(t)-u^{n}(t)\right|^{2}+\alpha E \int_{0}^{t}\left\|u^{n+1}(s)-u^{n}(s)\right\|^{2} d s \\
& \leq \frac{3 \alpha}{4} E \int_{0}^{t}\left\|u^{n+1}(s)-u^{n}(s)\right\|^{2} d s+\frac{\lambda^{2} \beta^{2}}{\alpha} \int_{0}^{t} \sup _{0 \leq \theta \leq s} E\left|u^{n}(\theta)-u^{n-1}(\theta)\right|^{2} d s \\
&+\frac{4}{\alpha} C^{F_{1}} \int_{0}^{t} \sup _{0 \leq \theta \leq s} E\left|u^{n}(\theta)-u^{n-1}(\theta)\right|^{2} d s \\
&+\left(\frac{3 K_{G_{1}}}{\alpha}+1\right) C^{G_{0}} \int_{0}^{t} \sup _{0 \leq \theta \leq s} E\left|u^{n}(\theta)-u^{n-1}(\theta)\right|^{2} d s,
\end{aligned}
$$

where $\beta>0$ is the constant such that $|v| \leq \beta\|v\| \forall v \in V$. Consequently, (3.18) yields

$$
\begin{gathered}
\sup _{0 \leq s \leq t} E\left|u^{n+1}(s)-u^{n}(s)\right|^{2}+\frac{\alpha}{4} E \int_{0}^{t}\left\|u^{n+1}(s)-u^{n}(s)\right\|^{2} d s \\
\leq k \int_{0}^{t} \sup _{0 \leq \theta \leq s} E\left|u^{n}(\theta)-u^{n-1}(\theta)\right|^{2} d s,
\end{gathered}
$$

for all $t \in[0, T]$ and all $n \geq 1$, where $k=\frac{2 \lambda^{2} \beta^{2}}{\alpha}+\frac{8}{\alpha} C^{F_{1}}+2\left(\frac{3 K_{G_{1}}}{\alpha}+1\right) C^{G_{0}}$. Now, if we denote

$$
\rho^{n}(t)=\sup _{0 \leq s \leq t} E\left|u^{n+1}(s)-u^{n}(s)\right|^{2}+\frac{\alpha}{4} E \int_{0}^{t}\left\|u^{n+1}(s)-u^{n}(s)\right\|^{2} d s, \forall n \geq 1, \forall t \in[0, T],
$$

we can deduce from (3.19) that

$$
\rho^{n}(t) \leq \frac{(k T)^{n-1}}{(n-1) !} \rho^{1}(T), \forall n \geq 1, \forall t \in[0, T]
$$

and thus, $\forall n \geq 1$,

$$
\sup _{0 \leq s \leq T} E\left|u^{n+1}(s)-u^{n}(s)\right|^{2}+\frac{\alpha}{4} E \int_{0}^{T}\left\|u^{n+1}(s)-u^{n}(s)\right\|^{2} d s \leq \frac{(k T)^{n-1}}{(n-1) !} \rho^{1}(T),
$$

and, in particular, $\left\{u^{n}\right\}_{n \geq 1}$ is a Cauchy sequence in $I^{2}(-h, T ; V)$.

Now, in order to prove that $\left\{u^{n}\right\}_{n>1}$ is a Cauchy sequence in $L^{2}(\Omega ; C(-h, T ; H))$, we consider again (3.17), take $\sup _{0 \leq s \leq T}$ and, finally, expectation, so that we obtain

$$
E\left(\sup _{0 \leq s \leq T}\left|u^{n+1}(s)-u^{n}(s)\right|^{2}\right)
$$




$$
\begin{aligned}
\leq & |\lambda| E \int_{0}^{T}\left|\left(u^{n+1}(s)-u^{n}(s), u^{n}(s)-u^{n-1}(s)\right)\right| d s \\
& +2 E \int_{0}^{T}\left|\left\langle F_{1}\left(s, u_{s}^{n}\right)-F_{1}\left(s, u_{s}^{n-1}\right), u^{n+1}(s)-u^{n}(s)\right\rangle\right| d s \\
& +2 E \int_{0}^{T} \mid\left(G_{0}\left(s, u_{s}^{n}\right)-G_{0}\left(s, u_{s}^{n-1}\right), G_{1}\left(s, u_{s}^{n+1}\right)-G_{1}\left(s, u_{s}^{n}\right) \mid d s\right. \\
& +2 E\left(\sup _{0 \leq s \leq T} \int_{0}^{s}\left(G_{0}\left(\theta, u_{\theta}^{n}\right)-G_{0}\left(\theta, u_{\theta}^{n-1}\right), u^{n+1}(\theta)-u^{n}(\theta)\right) d W(\theta)\right) \\
& +2 E\left(\sup _{0 \leq s \leq T} \int_{0}^{s}\left(G_{1}\left(\theta, u_{\theta}^{n+1}\right)-G_{1}\left(\theta, u_{\theta}^{n}\right), u^{n+1}(\theta)-u^{n}(\theta)\right) d W(\theta)\right) \\
& +E \int_{0}^{T}\left|G_{0}\left(s, u_{s}^{n}\right)-G_{0}\left(s, u_{s}^{n-1}\right)\right|^{2} d s
\end{aligned}
$$

for all $t \in[0, T]$ and all $n \geq 1$.

Using $\left(F_{1} .4\right)$, we obtain

$$
\begin{aligned}
& 2 E \int_{0}^{T}\left|\left\langle F_{1}\left(s, u_{s}^{n}\right)-F_{1}\left(s, u_{s}^{n-1}\right), u^{n+1}(s)-u^{n}(s)\right\rangle\right| d s \\
& \quad \leq C^{F_{1}} \int_{0}^{T} \sup _{0 \leq \theta \leq s} E\left|u^{n}(\theta)-u^{n-1}(\theta)\right|^{2} d s+E \int_{0}^{T}\left\|u^{n+1}(s)-u^{n}(s)\right\|^{2} d s .
\end{aligned}
$$

From Burkholder-Davis-Gundy's inequality, $\left(G_{0} .4\right)$ and $\left(G_{1} .4\right)$, we have

$$
\begin{aligned}
& 2 E\left(\sup _{0 \leq s \leq T} \int_{0}^{s}\left(G_{0}\left(\theta, u_{\theta}^{n}\right)-G_{0}\left(\theta, u_{\theta}^{n-1}\right), u^{n+1}(\theta)-u^{n}(\theta)\right) d W(\theta)\right) \\
& \leq \frac{1}{3} E\left(\sup _{0 \leq s \leq T}\left|u^{n+1}(s)-u^{n}(s)\right|^{2}\right)+27 C^{G_{0}} \int_{0}^{T} \sup _{0 \leq \theta \leq s} E\left|u^{n}(\theta)-u^{n-1}(\theta)\right|^{2} d s,\left(3.2{ }^{2}\right. \\
& E \int_{0}^{T}\left|G_{0}\left(s, u_{s}^{n}\right)-G_{0}\left(s, u_{s}^{n-1}\right)\right|^{2} d s \leq C^{G_{0}} \int_{0}^{T} \sup _{0 \leq \theta \leq s} E\left|u^{n}(\theta)-u^{n-1}(\theta)\right|^{2} d s \\
& 2 E \int_{0}^{T} \mid\left(G_{0}\left(s, u_{s}^{n}\right)-G_{0}\left(s, u_{s}^{n-1}\right), G_{1}\left(s, u_{s}^{n+1}\right)-G_{1}\left(s, u_{s}^{n}\right) \mid d s\right. \\
& \leq C^{G_{0}} \int_{0}^{T} \sup _{0 \leq \theta \leq s} E\left|u^{n}(\theta)-u^{n-1}(\theta)\right|^{2} d s+K_{G_{1}} E \int_{0}^{T}\left\|u^{n+1}(s)-u^{n}(s)\right\|^{2} d s
\end{aligned}
$$

and

$$
\begin{aligned}
& 2 E\left(\sup _{0 \leq s \leq T} \int_{0}^{s}\left(G_{1}\left(\theta, u_{\theta}^{n+1}\right)-G_{1}\left(\theta, u_{\theta}^{n}\right), u^{n+1}(\theta)-u^{n}(\theta)\right) d W(\theta)\right) \\
& \quad \leq \frac{1}{3} E\left(\sup _{0 \leq s \leq T}\left|u^{n+1}(s)-u^{n}(s)\right|^{2}\right)+27 K_{G_{1}} E \int_{0}^{T}\left\|u^{n+1}(s)-u^{n}(s)\right\|^{2} d s .
\end{aligned}
$$

Also,

$$
\begin{aligned}
& |\lambda| E \int_{0}^{T}\left(u^{n+1}(s)-u^{n}(s), u^{n}(s)-u^{n-1}(s)\right) d s \\
& \leq \frac{1}{2} E \int_{0}^{T}\left\|u^{n+1}(s)-u^{n}(s)\right\|^{2} d s+\frac{\lambda^{2} \beta^{2}}{2} \int_{0}^{T} \sup _{0 \leq \theta \leq s} E\left|u^{n}(\theta)-u^{n-1}(\theta)\right|^{2} d s .
\end{aligned}
$$


From (3.20)-(3.27), we deduce that $\left\{u^{n}\right\}_{n \geq 1}$ is a Cauchy sequence in $L^{2}(\Omega ; C(-h, T ; H))$. Thus, there exists $u$ such that $u^{n} \rightarrow u$ in $I^{2}(-h, T ; V) \cap L^{2}(\Omega ; C(-h, T ; H))$. Now, by a similar argument to the one in the proof of theorem 2.1, we can deduce that $u$ is the solution of problem $(P)$

Remark 3.1. The hypothesis concerning the compactness of the injection $V \subset H$ can be omitted if, for example, $\psi \equiv 0$.

Remark 3.2. Theorems 2.1. and 3.1. can be extended to the case in which $W(t)$ is an $\mathbb{R}^{n}$-valued (or Hilbert valued) Wiener process.

\section{An example}

To illustrate our theory, mainly Theorem 3.1, we shall consider the following situation, which cannot be handled with the results in Caraballo [2] or Caraballo et al. [4].

Assume $\mathcal{O} \subset \mathbb{R}^{n}$ is a bounded open set. Let us set $H=L^{2}(\mathcal{O}), V=H_{0}^{1}(\mathcal{O})$ and $V^{*}=H^{-1}(\mathcal{O})$.

Let $\phi:[0, T] \times \mathbb{R}^{n} \rightarrow \mathbb{R}^{n}$ be a continuous map such that there exists $c_{\phi}>0$ such that $|\phi(t, x)|_{\mathbf{R}^{n}} \leq c_{\phi}|x|_{\mathbf{R}^{n}}$ for all $(t, x) \in[0, T] \times \mathbb{R}^{n}$, and suppose that

$$
(\phi(t, x)-\phi(t, y)) \cdot(x-y) \leq 0 \forall t \in[0, T], \forall x, y \in \mathbb{R}^{n},
$$

where we denote by $\cdot$ the escalar product in $\mathbb{R}^{n}$. It is easy to see that the family of operators $A(t, \cdot)$ defined by

$$
\langle A(t, u), v\rangle=-\int_{\mathcal{O}} \nabla u(x) \cdot \nabla v(x) d x-\int_{\mathcal{O}} \phi(t, \nabla u(x)) \cdot \nabla v(x) d x \forall t \in[0, T], \forall u, v \in V,
$$

satisfies hypotheses (A.1)-(A.4), with $\lambda=0$ and $\alpha \leq 2$.

Let us consider now a measurable map $k_{1}:[0, T] \times \mathbb{R} \rightarrow \mathbb{R}^{n}$ and a measurable function $\omega_{1}:[0, T] \rightarrow \mathbb{R}$ such that $0 \leq \omega_{1}(t) \leq h$ for all $t \in[0, T]$. Suppose that $k_{1}(t, 0)=0, \forall t \in[0, T]$, and that there exists $L_{k_{1}}>0$ such that

$$
\left|k_{1}(t, a)-k_{1}(t, b)\right|_{\mathbf{R}^{n}} \leq L_{k_{1}}|a-b|, \forall t \in[0, T], \forall a, b \in \mathbb{R} .
$$

Denote by $F_{1}(t, \cdot)$ the family of operators defined by

$$
\left\langle F_{1}(t, \xi), v\right\rangle=-\int_{\mathcal{O}} k_{1}\left(t, \xi\left(-\omega_{1}(t)\right)(x)\right) \cdot \nabla v(x) d x, \forall \xi \in C(-h, 0 ; H), \forall v \in V,
$$

for each $t \in[0, T]$. $L_{k_{1}}^{2}$.

Then, the family $F_{1}(t, \cdot)$ satisfies assumptions $\left(F_{1} .1\right)-\left(F_{1} .4\right)$, with $C_{F_{1}}=L_{k_{1}}^{2}$ and $C^{F_{1}}=$

Consider also $k_{2}:[0, T] \times \mathbb{R}^{n} \rightarrow \mathbb{R}^{n}$, measurable, and $\omega_{2} \in C^{1}([0, T])$ such that $0 \leq$ $\omega_{2}(t) \leq h$ for all $t \in[0, T]$, and $\omega_{2}^{*}=\max _{t \in[0, T]} \omega_{2}^{\prime}(t)<1$. Suppose that $k_{2}(t, 0)=0, \forall t \in$ $[0, T]$, and that there exists $L_{k_{2}}>0$ such that

$$
\left|k_{2}(t, x)-k_{2}(t, y)\right|_{\mathbf{R}^{n}} \leq L_{k_{2}}|x-y|_{\mathbf{R}^{n}}, \forall t \in[0, T], \forall x, y \in \mathbb{R}^{n} .
$$


Denote by $F_{2}(t, \cdot)$ the family of operators defined by

$$
\left\langle F_{2}(t, \xi), v\right\rangle=-\int_{\mathcal{O}} k_{2}\left(t, \nabla \xi\left(-\omega_{2}(t)\right)(x)\right) \cdot \nabla v(x) d x, \forall \xi \in C(-h, 0 ; V), \forall v \in V,
$$

for each $t \in[0, T]$. Then, the family $F_{2}(t, \cdot)$ satisfies hypotheses $\left(F_{2} \cdot 1\right)-\left(F_{2} .4\right)$, with $C_{F_{2}}=L_{k_{2}}^{2}$ and $K_{F_{2}}=\frac{L_{k_{2}}^{2}}{1-\omega_{2}^{*}}$.

Finally, let $l_{0}:[0, T] \times \mathbb{R} \rightarrow \mathbb{R}$ and $l_{1}:[0, T] \times \mathbb{R}^{n} \rightarrow \mathbb{R}$ be two measurable functions, such that $l_{0}(t, 0)=l_{1}(t, 0)=0$ for all $t \in[0, T]$, and there exist $L_{l_{0}}>0$ and $L_{l_{1}}>0$ such that

$$
\left|l_{0}(t, a)-l_{0}(t, b)\right| \leq L_{l_{0}}|a-b|, \forall t \in[0, T], \forall a, b \in \mathbb{R},
$$

and

$$
\left|l_{1}(t, x)-l_{1}(t, y)\right| \leq L_{l_{1}}|x-y|_{\mathbf{R}^{n}}, \forall t \in[0, T], \forall x, y \in \mathbb{R}^{n} .
$$

Let us also fix $\rho_{i}:[0, T] \rightarrow \mathbb{R}, i=0,1$, two measurable functions such that $0 \leq \rho_{i}(t) \leq h$ for all $t \in[0, T]$ and $i=0,1, \rho_{1} \in C^{1}([0, T])$, and $\rho_{1}^{*}=\max _{t \in[0, T]} \rho_{1}^{\prime}(t)<1$.

Then, if we define

$$
G_{0}(t, \xi)(x)=l_{0}\left(t, \xi\left(-\rho_{0}(t)\right)(x)\right), \forall t \in[0, T], \forall \xi \in C(-h, 0 ; H) \text {, a.e. } x \in \mathcal{O},
$$

and

$$
G_{1}(t, \xi)(x)=l_{1}\left(t, \nabla \xi\left(-\rho_{1}(t)\right)(x)\right), \forall t \in[0, T], \forall \xi \in C(-h, 0 ; V), \text { a.e. } x \in \mathcal{O},
$$

it is easy to check that $G_{0}$ satisfies $\left(G_{0} .1\right)-\left(G_{0} .4\right)$, and that $G_{1}$ satisfies hypotheses $\left(G_{1} .1\right)$ $\left(G_{1} .4\right)$, with $C_{G_{0}}=C^{G_{0}}=L_{l_{0}}^{2}, C_{G_{1}}=L_{l_{1}}^{2}$, and $K_{G_{1}}=\frac{L_{l_{1}}^{2}}{1-\rho_{1}^{*}}$.

As for hypothesis $(A .5)$, it is fulfilled with $\widehat{\lambda}$ large enough provided

$$
\frac{2 L_{k_{2}}}{\sqrt{1-\omega_{2}^{*}}}+\frac{L_{l_{1}}^{2}}{1-\rho_{1}^{*}}<2
$$

Consequently, under all the hypotheses above, we can ensure that given $\psi \in I^{2}\left(-h, 0 ; H_{0}^{1}(\mathcal{O})\right) \cap$ $L^{2}\left(\Omega ; C\left(-h, 0 ; L^{2}(\mathcal{O})\right)\right), f \in I^{2}\left(0, T ; H^{-1}(\mathcal{O})\right)$, and $g \in I^{2}\left(0, T ; L^{2}(\mathcal{O})\right)$, there exists a unique solution $u \in I^{2}\left(-h, T ; H_{0}^{1}(\mathcal{O})\right) \cap L^{2}\left(\Omega ; C\left(-h, T ; L^{2}(\mathcal{O})\right)\right)$ to the corresponding problem $(P)$. Such a solution, satisfies, in a generalized sense, the problem

$$
\left\{\begin{array}{l}
\frac{\partial u(t)}{\partial t}=\Delta u(t)+\nabla \cdot(\phi(t, \nabla u(t)))+\nabla \cdot\left(k_{2}\left(t, \nabla u\left(t-\omega_{2}(t)\right)\right)\right)+\nabla \cdot\left(k_{1}\left(t, u\left(t-\omega_{1}(t)\right)\right)\right) \\
+f(t)+\left(l_{1}\left(t, \nabla u\left(t-\rho_{1}(t)\right)\right)+l_{0}\left(t, u\left(t-\rho_{0}(t)\right)\right)+g(t)\right) \frac{\partial W(t)}{\partial t} \quad \text { in } \mathcal{O} \times(0, T) \\
u(0)=0 \quad \text { on } \partial \mathcal{O} \times(0, T), \\
u(t)=\psi(t) \quad \text { in } \mathcal{O} \times[-h, 0]
\end{array}\right.
$$

where, for a vector function $\vec{v}=\left(v_{1}, \ldots, v_{n}\right)$ defined on $\mathcal{O}$, we denote by $\nabla \cdot \vec{v}$ the divergence of $\vec{v}$ defined by $\nabla \cdot \vec{v}=\sum_{i=1}^{n} \frac{\partial v_{i}}{\partial x_{i}}$. 
Acknowledgement. This work has been partly supported by Junta de Andalucia Project FQM314.

\section{References}

[1] M. Artola, Equations et inequations variationnelles a retard, Ann. Sc. Math. Québec vol. I, no. 2 (1977), 131-152.

[2] T. Caraballo, Existence and Uniqueness of Solutions for non-linear Stochastic Partial Differential Equations, Collectanea Mathematica 42(1) (1991), 51-74.

[3] T. Caraballo, Nonlinear Partial Functional Differential Equations: Existence and Stability, J. Math. Anal. Appl., to appear.

[4] T. Caraballo, K. Liu and A. Truman, Stochastic Functional Partial Differential Equations: Existence, Uniqueness and Asymptotic Decay Property, Proc. R. Soc. Lond. A 456 (2000), 1775-1802.

[5] G. Da Prato and J. Zabczyk, Stochastic Equations in Infinite Dimensions, Cambridge (1992).

[6] E. Pardoux, Équations aux Dérivées Partielles Stochastiques Non Linéaires Monotones, Thèse, Université Paris XI (1975).

[7] E. Pardoux, Stochastic Partial Differential Equations and Filtering of Diffusion Processes, Stochastic, 3 (1979), 127-167.

[8] J. Real, Contribución al Estudio de una Clase de Ecuaciones en Derivadas Parciales Estocásticas con Retardo, Thesis, University of Sevilla (1980).

[9] J. Real, Stochastic Partial Differential equations with Delays, Stochastics 8 (1982-1983), 81-102.

[10] W. Ruess, Existence of solutions to partial functional differential equations with delay, in Theory and Applications of Nonlinear Operators of Accretive and Monotone Type, (ed. A.G. Kartsatos), Marcel-Dekker, Lecture Notes Pure Appl. Math. 178, New York (1996), $259-288$.

[11] J. Wu, Theory and applications of partial functional differential equations, SpringerVerlag, New York (1996). 